\title{
Growth-dependent effect of muscleblind knockdown on Caenorhabditis elegans
}

\author{
Li-Chun Wang ${ }^{\text {a }}$, Wan-Tzu Hung ${ }^{\text {a }}$, Huichin Pan ${ }^{\mathrm{a}, *}$, Kuan-Yu Chen ${ }^{\mathrm{a}}$, Yi-Chun Wu ${ }^{\mathrm{b}}$, \\ Yu-Fan Liu ${ }^{a}$, Kuang-Ming Hsiao ${ }^{\text {a,* }}$ \\ a Department of Biomedical Sciences, Chung Shan Medical University, Taichung, Taiwan, ROC \\ ${ }^{\mathrm{b}}$ Institute of Molecular and Cellular Biology, National Taiwan University, Taipei, Taiwan, ROC
}

Received 8 November 2007

Available online 17 December 2007

\begin{abstract}
The muscleblind-like (MBNL) proteins are tissue-specific alternative splicing regulators. Dysfunction of MBNL has been implicated in the pathogenesis of expanded CUG repeats-associated myotonic dystrophy (DM). In this study, we describe the identification and functional characterization of a Caenorhabditis elegans muscleblind $(\mathrm{CeMbl})$ gene. $\mathrm{CeMbl}$ is a single gene alternatively spliced to generate two isoforms (CeMBL-A and CeMBL-B). It displays a high homology with human MBNL1 in the C3H1 zinc finger domains. CeMbl transcripts are detected in larval and adult stages. However, inactivation of $\mathrm{CeMbl}$ by RNA-mediated interference results in muscle phenotype only at adulthood. Immunofluorescence staining using anti-vinculin antibody reveals that the organization of dense body is disrupted in affected worms. Our results demonstrate a growth-dependent requirement of $\mathrm{CeMbl}$ on muscle structure and function. They also provide a possible molecular basis for the developmentally regulated toxicity of expanded CUG repeats.
\end{abstract}

(c) 2007 Elsevier Inc. All rights reserved.

Keywords: CeMbl; RNAi; CUG repeats; Dense body; C. elegans

Myotonic dystrophy (dystrophia myotonia, DM) is a dominantly inherited form of muscular disorder caused by two different microsatellite expansions. Type 1 (DM1) is caused by an expansion of CTG repeats in the 3'untranslated region of the $D M P K$ gene [1]. In normal population, the repeat number ranges in size from 5 to 37 . The number ranges from 50 to several thousand at disease loci. Type 2 (DM2) is caused by a CCTG repeat expansion in intron 1 of the ZNF9 gene [2]. While most DM patients present clinical manifestations at adulthood, congenital cases have been found only in DM1 with large CTG repeat expansion (usually $>1000$ repeats) [3].

\footnotetext{
* Corresponding authors. Address: Department of Biomedical Sciences, Chung Shan Medical University, No. 110, Section 1, Jinguo North Road, Taichung, Taiwan, ROC. Fax: +886 423248109.

E-mail addresses: hp29@csmu.edu.tw (H. Pan), kmh@csmu.edu.tw (K.-M. Hsiao).
}

The RNA transcribed from the expanded alleles containing CTG or CCTG repeats are proposed to compromise the regulation of alternative splicing by altering the functions of RNA-binding proteins [4]. Two families of RNA-binding proteins, CELF (CUG-BP and ETR-3-like factors) and muscleblind-like (MBNL), have been implicated in DM pathogenesis. The CELF family includes CUG-BP1 and the related protein ETR-3/CUG-BP2. CUG-BP1 does not bind efficiently to expanded RNA CUG repeats [5] and does not colocalize with the nuclear foci of expanded RNA [6,7]. However, both the activity and steady-state levels of CUG-BP1 are increased in DM1 muscle [8]. In addition, over-expression of CUGBP1 in mice reproduces DM pathological features and leads to the DM splicing pattern for cardiac troponin T (Tnnt2), myotubularin-related 1 gene (Mtmr 1), and chloride channel 1 (Clcnl) in striated muscle tissue [9]. Muscleblind was initially identified as a protein required for 
photoreceptor and muscle differentiation in Drosophila $[10,11]$. Its mammalian homologs were subsequently found to function as regulators of alternative splicing [12]. Human muscleblind-like proteins consist of three members (MBNL1, 2, and 3), and all have been shown to bind to the nuclear foci of expanded repeats [13]. The observations that Mbnll knockout mice reproduce muscle, eye, and splicing abnormalities that are characteristic of DM disease [14] and that Mbnll over-expression may reverse myotonia and missplicing in a mouse poly(CUG) model further confirms the role of this protein in DM pathogenesis [15].

Recently, we have shown that the CUG repeats caused a length-dependent toxicity in Caenorhabditis elegans, including muscle abnormality at adulthood (CUG125) and growth arrest during embryogenesis (CUG213) [16]. Inactivation of etr-1, a C. elegans homolog of mammalian CUG$\mathrm{BP} 1$, by RNA-mediated interference (RNAi) resulted in embryonic lethality [17]. Whereas in a full-genome RNAi study, knockdown of the predicted $C$. elegans muscleblind $(\mathrm{CeMbl})$ gene $(\mathrm{K} 02 \mathrm{H} 8.1)$ did not result in any phenotype in early embryogenesis [18]. To further elucidate the role of CeMBL in CUG repeats-mediated toxicity, we have here cloned the $C e M b l$ cDNA and analyzed the effect of RNAi targeted to $\mathrm{CeMbl}$ transcripts. Our results demonstrate that inactivation of $\mathrm{CeMbl}$ results in a growth-dependent muscle phenotype similar to that caused by CUG125.

\section{Materials and methods}

cDNA cloning and Accession numbers. To clone the cDNA encoding CeMBL and ETR-1 proteins, total RNA was isolated from mixed populations of C. elegans (Bristol N2) using TRI-reagent (Molecular Research Center). Reverse transcription-polymerase chain reaction (RT-PCR) was performed using SuperScript II RT (Invitrogen) according to the manufacturer's instructions. The primer sets used to amplify $\mathrm{CeMbl}$ and etr-1 cDNA were $m b l-F 1$ (5'-CCAGCTAGCAGAATGTTCGACGAAAAC AG-3')/mbl-R2 (5'-CAGCCATGGAATGTGTGTCTAGAATGG-3') and etr-1-F1 (5'-GCTAGCCAGTTCGAGTGGTACCA-3')/etr-1-R1 (5'CCATGGGATACTTTTATCGATTG-3'), respectively. The identified cDNA sequences have been deposited at the GenBank under Accession Nos. EF520300 (CeMbl-a), EF520301 (CeMbl-b), EF523766 (etr-1a), EF523767 (etr-1b), EF523768 (etr-1c), EF523769 (etr-1d), and EF523770 (etr-1e).

Gene structure analysis. Gene structures of $\mathrm{CeMbl}$ and etr-1 (shown in Fig. 1 and Supplementary Fig. 1, respectively) were determined using BlastN by comparing the cDNA sequences with the corresponding genes (T01D1.2 and K02H8.1 for etr-1 and $\mathrm{CeMbl}$, respectively) at Wormbase (http://www.wormbase.org/).

Plasmids. L4440 (pPD129.36) plasmid was used as a feeding vector for RNAi assay. To prepare the construct for $\mathrm{CeMbl}$ (RNAi), a 533-bp DNA fragment (spanning from exons 1 to 4 ) obtained from PCR amplification of CeMbl-a cDNA using primers, $m b l-F 1$ 5'-CCAGCTAGCAGAA TGTTCGACGAAAACAG-3' and $m b l-R l$ 5'-CACTGCCGCTGCT GTATAAG-3', was first cloned into pGEMT-easy vector and then transferred into L4440 plasmid. The feeding vector for etr-1 (RNAi) contains a 864-bp DNA fragment (spanning from exons 3 to 10) obtained from PCR amplification of etr-1a cDNA using primers etr-1-F2 $5^{\prime}-\mathrm{G}$ GAATTCGAGGAGAATCTGCGTGAC-3' and etr-1-R2 $5^{\prime}$-GGAA TTCTGTTGATGATTGGCGAGCG- $3^{\prime}$. Successful construction of plasmids was confirmed by DNA sequencing.

RNA-mediated interference. To perform RNAi, transformed bacteria HT115 (DE3) containing the RNAi plasmids served as food source. The
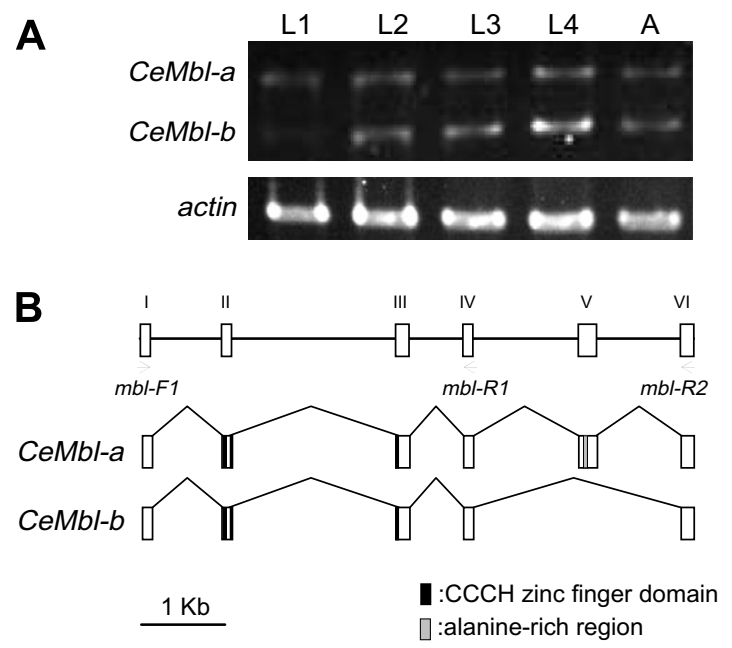

Fig. 1. C. elegans muscleblind gene expression and its protein products. (A) $\mathrm{CeMbl}$ expression during worm development. RNA extracted from worms at distinct developmental stages, including L1-L4 and adult stage (indicated as A), were subjected to RT-PCR. The PCR products were then analyzed using electrophoresis. (B) Genomic organization and gene structure of $\mathrm{CeMbl}$. Exons are depicted as boxes and introns as lines. The locations of primers used to amplify the whole $\mathrm{CeMbl}$ cDNA ( $\mathrm{CeMbl}$ $F 1 / R 2)$ and the cDNA fragment for RNAi assay (CeMbl-Fl/Rl) are indicated. Two CeMbl transcripts, designated as CeMbl- $a$ and CeMbl-b, were identified in this study. The scale bar corresponds to $1 \mathrm{~kb}$. The nucleotide sequences encoding two zinc finger $(\mathrm{C} 3 \mathrm{H})$ domains and one alanine-rich domain are found in exons $2-3$ and 5, respectively.

expression of double-stranded RNA was induced by IPTG. For morphology, life span, and muscle phenotype analysis, freshly prepared synchronized eggs were cultured on IPTG plate for $36 \mathrm{~h}$ and then transferred to standard NGM plate. For brood size and embryogenesis analysis, the eggs were cultured on the IPTG-containing plate until the next generation was produced.

Phenotypes. Morphological examination was performed by using a Zeiss Axioskop 2 microscope. The musculature was examined by phalloidin staining as previously described [16]. To examine the locomotory behavior, worms were picked to fresh spread plates and the moving tracks were recorded after $30 \mathrm{~min}$ by using an Olympus SZX12 dissection microscope. Motility is calculated as the number of regular S-pattern body bends performed in $3 \mathrm{~min}$ at $25^{\circ} \mathrm{C}$. Life span was defined as the interval between the time the animal was hatched and the time it died (that is, it stopped moving for more than $1 \mathrm{~h}$ and did not respond to contact stimulation).

Immunofluorescence staining. Whole worms were fixed by Bouin's fixative according to a protocol described previously [19]. The fixed worms were blocked in AbA solution ( $1 \times$ PBS, $1.0 \%$ BSA, $0.5 \%$ Triton X-100, and $10 \mathrm{mM}$ NaAzide) and then incubated with anti-vinculin antibody (Santa Cruz, 1:200 dilution in $\mathrm{AbA}$ ) at $4{ }^{\circ} \mathrm{C}$ overnight, followed by three washes in PBST. After incubation with TR-conjugated secondary antibody (Santa Cruz, 1:200 dilution in AbA) for $6 \mathrm{~h}$ at room temperature, the worms were washed and the dense body alignment was visualized under a fluorescence microscope (Zeiss Axioplan).

\section{Results and discussion}

\section{Two alternatively spliced forms of CeMbl transcripts}

All invertebrate genomes analyzed, including C. elegans, contain a single muscleblind gene [20]. To clone the $\mathrm{CeMbl}$ gene, a predicted $\mathrm{CeMbl}$ gene (K02H8.1) was first retrieved 
by sequence similarity searches against NCBI nonredundant (nr) database using Drosophila melanogaster muscleblind-A (NP_788392.1) as a query. Subsequently, RTPCR was performed to amplify the CeMbl cDNA. Two alternatively spliced isoforms, CeMbl- $a$ and CeMbl-b, were identified. Both isoforms are expressed in larval and adult stages (Fig. 1A). An examination of the gene structure reveals that the CeMbl-a cDNA contains six exons (I-VI) whereas $C e M b l-b$ cDNA contains only five exons with exclusion of exon V (Fig. 1B).

The encoded proteins from CeMbl- $a$ and $C e M b l-b$ transcripts (designated as CeMBL-A and CeMBL-B, respectively) both contain two tandem zinc finger domains (Supplementary Fig. 2). Each zinc finger is composed of three cysteine and one histidine residues (known as $\mathrm{CCCH}$ domain). This structural domain is present in all muscleblind proteins and is required for binding to pre-mRNA [20]. The deduced amino acid sequences in the $\mathrm{CCCH}$ domain of CeMBL showed approximately $70-90 \%$ similarity (60-80\% identity) to the MBNL orthologs in other species (Supplementary Fig. 2). However, the homology is low in the rest part of the protein. Apart from the characteristic $\mathrm{CCCH}$ zinc finger domains, an alanine-rich region was found in CeMBL-A, but not in CeMBL-B (Fig. 1B). The alanine-rich region has been shown to be required for high-affinity binding of AUF1 (an RNA-binding protein) to its target sequence $(\mathrm{A}+\mathrm{U}$-rich elements) and the region also contributed to the dimerization of this protein [21]. Whether the alanine-rich region plays a role in binding to RNA or in protein-protein interaction of CeMBL isoforms needs further verification.

\section{CeMBL is not required for embryogenesis and larval development}

To investigate the function of CeMBL, RNAi experiments were performed by feeding method. Worms fed with empty vector-containing bacteria were used as a control. In parallel, RNAi targeted to etr-l was performed for comparison. As shown in Fig. 2, control (RNAi) treatment did not cause any effect on worm body shape until day 7 . When the animals aged $(\geqslant 10$ days old), approximately $20 \%$ of them displayed abnormal morphology, a ratio slightly higher than that in untreated population. The effect of etr-l (RNAi) on larval development was noticed as early as in the period of RNAi treatment. The affected worms presented distorted body shapes (upper panel in Fig. 2). The detrimental effect became more prominent as they grew older (Fig. 2). The average life span of the etr-1 (RNAi) worms was significantly shorter than that of controls $(6.9 \pm 0.4$ vs $16.3 \pm 0.7$ days, Fig. 3D). It was previously shown that the embryos of etr-1 (RNAi) worms remained encased in the egg shells or were paralyzed after hatching and never showed further development [17]. Consistently, we found that etr-1 (RNAi) worms had a brood size significantly smaller than control group


Fig. 2. Morphological abnormality of CeMbl (RNAi) worms. (Upper panel) Representative pictures of body shape of etr-1 (RNAi), CeMbl (RNAi), and control (RNAi) worms. (Lower panel) Growth-dependent effect of RNAi treatment on worm morphology. The number of examined animals for etr-1 (RNAi), CeMbl (RNAi), and control (RNAi) worms is 44,43 , and 30, respectively. The period of RNAi treatment is indicated.

(Fig. 3A). This could have resulted from defective embryogenesis (Fig. 3B). In contrast, the effect of $\mathrm{CeMbl}$ (RNAi) on morphology was neither observed in larva nor in young adults ( $\leqslant 3$ days). Instead, the phenotype was present in the population at an age of 5 days or older (Fig. 2). A major feature of affected worms is a swollen protuberance occurring at the middle-posterior ventral part (around vulva) of the body (Fig. 2). Although $\mathrm{CeMbl}$ (RNAi) worms had a slightly smaller brood size (Fig. 3A), most of their embryos developed normally (Fig. 3B). This result indicates that etr-1 and $\mathrm{CeMbl}$ function at distinct developmental stages. Although $\mathrm{CeMbl}$ transcripts are present in larva (Fig. 1A), they appear to be not critical for early muscle development.

\section{Disrupted muscle structure and function in CeMbl (RNAi) adult worms}

Disruption of the mouse Mbnll gene was shown to cause muscle phenotypes characteristic of DM [14]. Recently, we have demonstrated that expanded CUG repeats are deleterious to body wall muscle of the worms in a length- and growth-dependent manner. To investigate if depletion of $\mathrm{CeMbl}$ is detrimental to C. elegans muscle, we examined the motility rate, locomotion behavior, and muscle structure of the RNAi-treated worms. At larval 

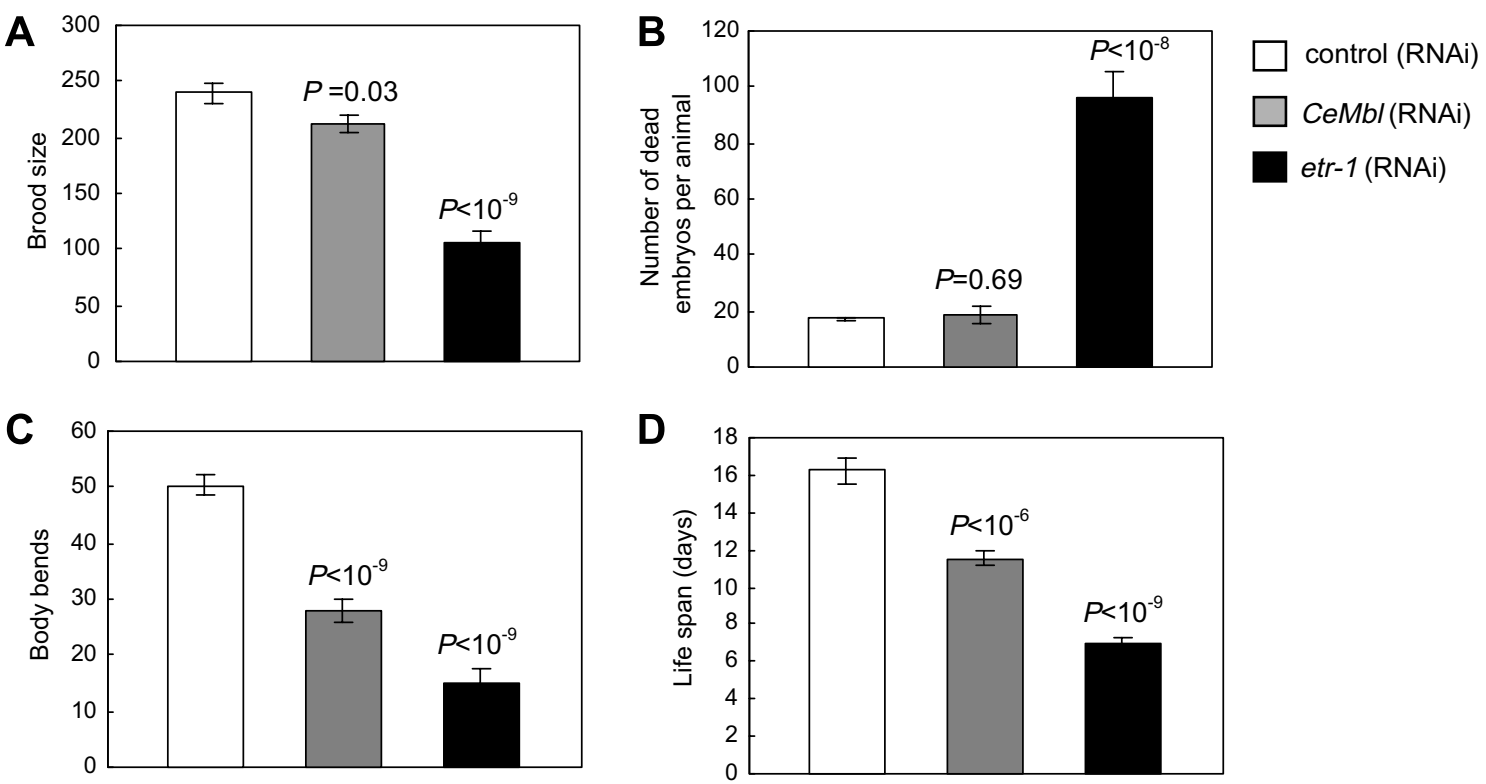

Fig. 3. Phenotypes of RNAi-treated C. elegans. Four phenotypes, including brood size (A), number of growth-arrested embryos (B), motility (C), and life span (D) of control (RNAi), CeMbl (RNAi), and etr-l (RNAi) worms were examined. At least 30 worms were analyzed for each phenotype. The effect of $\mathrm{CeMbl}$ (RNAi) and etr-1 (RNAi) was compared with that of control (RNAi) by using student's $t$-test.

stages, these animals displayed a locomotion pattern of regular S shapes. However, irregular track of movement was frequently left on plate by the adult worms (4-dayold) (upper panel in Fig. 4A). This suggests an age-dependent impairment of muscle coordination in these worms. In addition, the motility rate of the CeMbl (RNAi) worms was significantly reduced (Fig. 3C). Further examination

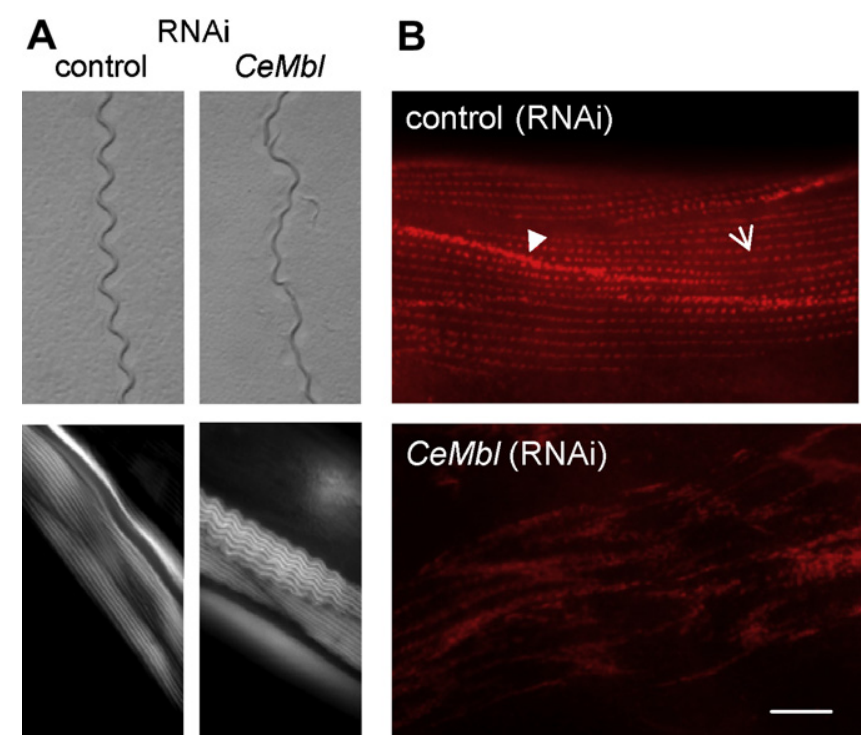

Fig. 4. Disrupted muscle function and structure in $\mathrm{CeMbl}$ (RNAi) $\mathrm{C}$. elegans. (A) Locomotory behavior (upper panels) and muscle morphology (lower panels). (B) Dense body alignment in muscle of control (RNAi) and $\mathrm{CeMbl}$ (RNAi) worms. Anti-DEB-1/vinculin staining of dense bodies was shown as red spots. Arrow indicates the dense body within a muscle cell. Arrowhead indicates the dense body at the cell boundary. The dense body structure is disrupted in the $\mathrm{CeMbl}$ (RNAi) animals. Bar, $10 \mu \mathrm{m}$. of the musculature by staining animals with the filamentous actin (F-actin) marker phalloidin revealed disrupted, wave-like structure in the body wall muscle cells (lower panel in Fig. 4A). The muscle phenotypes of $\mathrm{CeMbl}$ (RNAi) worms were similar to those observed in CUG125 worms [16], suggesting that the expanded CUG repeats may cause dominant effect on muscle by compromising the CeMBL function.

Disruption of Z-band structure is a common histological feature of muscle in DM patients and in flies deficient in muscleblind protein [22]. Like the Z-bands of higher eukaryotic muscle, the nematode dense bodies provide for side-to-side linkage of thin filaments as well as for linkage of oppositely oriented filaments. To investigate if the function of CeMBL on dense body organization has been evolutionarily conserved, animals of control and $\mathrm{CeMbl}$ (RNAi) were immunostained with an antibody against vinculin, a basal component of the dense body. As shown in Fig. 4B, the dense body in normal spindle-shaped muscle cells is aligned in an orderly pattern. It is also prominent at the cell-cell boundaries. In $\mathrm{CeMbl}$ (RNAi) muscle, this pattern of regular alignment is severely disrupted and the cell-cell boundaries become indistinguishable. The result indicates that CeMBL is required for the organization of dense body in C. elegans.

In summary, we have here identified two alternatively spliced forms of $\mathrm{CeMbl}$ transcripts and demonstrated that inactivation of $\mathrm{CeMbl}$ causes abnormal muscle structure and function in adult worms. The CUG125 worms also presented similar muscle phenotypes in a growth-dependent manner [16]. These observations suggest that expanded CUG repeats-induced toxicity on adults is mediated by compromising the CeMBL function. 


\section{Acknowledgments}

This work was supported by grants from the National Science Council, Taiwan (NSC 94-2320-B-040-012 and NSC 95-2320-B-040-002 to KMH, and NSC 91-2320-B040-034, NSC 92-2320-B-040-046 and NSC 93-2320-B040-054 to HP).

\section{Appendix A. Supplementary data}

Supplementary data associated with this article can be found, in the online version, at doi:10.1016/j.bbrc. 2007.12.024.

\section{References}

[1] M. Mahadevan, C. Tsilfidis, L. Sabourin, G. Shutler, C. Amemiya, G. Jansen, C. Neville, M. Narang, J. Barcelo, K. O'Hoy, et al., Myotonic dystrophy mutation: an unstable CTG repeat in the $3^{\prime}$ untranslated region of the gene, Science 255 (1992) 1253-1255.

[2] C.L. Liquori, K. Ricker, M.L. Moseley, J.F. Jacobsen, W. Kress, S.L. Naylor, J.W. Day, L.P. Ranum, Myotonic dystrophy type 2 caused by a CCTG expansion in intron 1 of ZNF9, Science 293 (2001) 864-867.

[3] L.P. Ranum, J.W. Day, Myotonic dystrophy: RNA pathogenesis comes into focus, Am. J. Hum. Genet. 74 (2004) 793-804.

[4] R.J. Osborne, C.A. Thornton, RNA-dominant diseases, Hum. Mol. Genet. 15 (Spec. No. 2) (2006) R162-R169.

[5] S. Michalowski, J.W. Miller, C.R. Urbinati, M. Paliouras, M.S Swanson, J. Griffith, Visualization of double-stranded RNAs from the myotonic dystrophy protein kinase gene and interactions with CUG-binding protein, Nucleic Acids Res. 27 (1999) 3534-3542.

[6] M. Fardaei, K. Larkin, J.D. Brook, M.G. Hamshere, In vivo colocalisation of MBNL protein with DMPK expanded-repeat transcripts, Nucleic Acids Res. 29 (2001) 2766-2771.

[7] H. Jiang, A. Mankodi, M.S. Swanson, R.T. Moxley, C.A. Thornton, Myotonic dystrophy type 1 is associated with nuclear foci of mutant RNA, sequestration of muscleblind proteins and deregulated alternative splicing in neurons, Hum. Mol. Genet. 13 (2004) 3079-3088.

[8] N.A. Timchenko, Z.J. Cai, A.L. Welm, S. Reddy, T. Ashizawa, L.T. Timchenko, RNA CUG repeats sequester CUGBP1 and alter protein levels and activity of CUGBP1, J. Biol. Chem. 276 (2001) 7820-7826.

[9] T.H. Ho, D. Bundman, D.L. Armstrong, T.A. Cooper, Transgenic mice expressing CUG-BP1 reproduce splicing mis-regulation observed in myotonic dystrophy, Hum. Mol. Genet. 14 (2005) $1539-1547$.

[10] G. Begemann, N. Paricio, R. Artero, I. Kiss, M. Perez-Alonso, M. Mlodzik, Muscleblind, a gene required for photoreceptor differentiation in Drosophila, encodes novel nuclear Cys3His-type zinc-fingercontaining proteins, Development 124 (1997) 4321-4331.
[11] R. Artero, A. Prokop, N. Paricio, G. Begemann, I. Pueyo, M. Mlodzik, M. Perez-Alonso, M.K. Baylies, The muscleblind gene participates in the organization of Z-bands and epidermal attachments of Drosophila muscles and is regulated by Dmef2, Dev. Biol. 195 (1998) 131-143.

[12] T.H. Ho, B.N. Charlet, M.G. Poulos, G. Singh, M.S. Swanson, T.A. Cooper, Muscleblind proteins regulate alternative splicing, EMBO J. 23 (2004) 3103-3112.

[13] M. Fardaei, M.T. Rogers, H.M. Thorpe, K. Larkin, M.G. Hamshere, P.S. Harper, J.D. Brook, Three proteins, MBNL, MBLL and MBXL, co-localize in vivo with nuclear foci of expanded-repeat transcripts in DM1 and DM2 cells, Hum. Mol. Genet. 11 (2002) 805-814.

[14] R.N. Kanadia, K.A. Johnstone, A. Mankodi, C. Lungu, C.A. Thornton, D. Esson, A.M. Timmers, W.W. Hauswirth, M.S. Swanson, A muscleblind knockout model for myotonic dystrophy, Science 302 (2003) 1978-1980.

[15] R.N. Kanadia, J. Shin, Y. Yuan, S.G. Beattie, T.M. Wheeler, C.A. Thornton, M.S. Swanson, Reversal of RNA missplicing and myotonia after muscleblind overexpression in a mouse poly(CUG) model for myotonic dystrophy, Proc. Natl. Acad. Sci. USA 103 (2006) 11748-11753.

[16] K.Y. Chen, H. Pan, M.J. Lin, Y.Y. Li, L.C. Wang, Y.C. Wu, K.M. Hsiao, Length-dependent toxicity of untranslated CUG repeats on Caenorhabditis elegans, Biochem. Biophys. Res. Commun. 352 (2007) 774-779.

[17] C.A. Milne, J. Hodgkin, ETR-1, a homologue of a protein linked to myotonic dystrophy, is essential for muscle development in Caenorhabditis elegans, Curr. Biol. 9 (1999) 1243-1246.

[18] B. Sonnichsen, L.B. Koski, A. Walsh, P. Marschall, B. Neumann, M. Brehm, A.M. Alleaume, J. Artelt, P. Bettencourt, E. Cassin, M. Hewitson, C. Holz, M. Khan, S. Lazik, C. Martin, B. Nitzsche, M. Ruer, J. Stamford, M. Winzi, R. Heinkel, M. Roder, J. Finell, H. Hantsch, S.J. Jones, M. Jones, F. Piano, K.C. Gunsalus, K. Oegema, P. Gonczy, A. Coulson, A.A. Hyman, C.J. Echeverri, Full-genome RNAi profiling of early embryogenesis in Caenorhabditis elegans, Nature 434 (2005) 462-469.

[19] M.L. Nonet, J.E. Staunton, M.P. Kilgard, T. Fergestad, E. Hartwieg, H.R. Horvitz, E.M. Jorgensen, B.J. Meyer, Caenorhabditis elegans rab-3 mutant synapses exhibit impaired function and are partially depleted of vesicles, J. Neurosci. 17 (1997) 8061-8073.

[20] M. Pascual, M. Vicente, L. Monferrer, R. Artero, The Muscleblind family of proteins: an emerging class of regulators of developmentally programmed alternative splicing, Differentiation 74 (2006) 6580.

[21] C.T. DeMaria, Y. Sun, L. Long, B.J. Wagner, G. Brewer, Structural determinants in AUF1 required for high affinity binding to A + Urich elements, J. Biol. Chem. 272 (1997) 27635-27643.

[22] L. Machuca-Tzili, H. Thorpe, T.E. Robinson, C. Sewry, J.D. Brook, Flies deficient in Muscleblind protein model features of myotonic dystrophy with altered splice forms of Z-band associated transcripts, Hum. Genet. 120 (2006) 487-499. 